\title{
Effect of Helicobacter pylori on cyclooxygenase-2 and inducible nitric oxide synthase in patients with gastric precancerous lesions and its clinical significance
}

\author{
HUI ZHANG* , CHUNSHENG DING* ${ }^{*}$ ZHIMIN SUO and YUHUA KANG \\ Department of Digestive Medicine, Henan University Huaihe Hospital, Kaifeng, Henan 475000, P.R. China
}

Received June 13, 2014; Accepted February 16, 2015

DOI: $10.3892 /$ etm.2015.2387

\begin{abstract}
The aim of this study was to investigate the effect of Helicobacter pylori (Hp) on cyclooxygenase-2 (COX-2) and inducible nitric oxide synthase (iNOS) levels in patients with gastric precancerous lesions and its clinical significance. A total of 114 patients with gastric precancerous lesions, 57 whom were positive for Hp (observation group) and 57 of whom were negative for Hp (control group), were selected for the study. The mRNA levels of COX-2 and iNOS in the gastric precancerous lesion tissue from the two groups of patients were analyzed through the reverse transcription-quantitative polymerase chain reaction (RT-qPCR). The protein expression levels of COX-2 and iNOS were analyzed using western blotting and an iNOS kit, respectively. In addition, normal human gastric mucosal GES-1 cells were cultured in vitro and stimulated by $\mathrm{Hp}$ for 3, 6, 9 and $12 \mathrm{~h}$. The variations in the mRNA and protein levels of COX-2 and iNOS were then analyzed via RT-qPCR and western blotting. Compared with the control group, the mRNA levels of COX-2 and iNOS in the gastric tissue from the observation group were significantly increased $(\mathrm{P}<0.05)$. Furthermore, the expression level of COX-2 and iNOS protein in the gastric tissue from the observation group was significantly higher than that in the tissue from the control group $(\mathrm{P}<0.05)$. In vitro analysis showed that the COX-2 and iNOS mRNA and protein levels were significantly increased in the Hp-stimulated normal human gastric mucosal GES-1 cells compared with those in the unstimulated cells. Furthermore, the effect was time-dependent $(\mathrm{P}<0.05)$. In conclusion, $\mathrm{COX}-2$ and iNOS are the main inflammatory markers. Hp can induce high expression levels of COX-2 and iNOS in gastric
\end{abstract}

Correspondence to: Dr Zhimin Suo, Department of Digestive Medicine, Henan University Huaihe Hospital, 115 Ximen Street, Kaifeng, Henan 475000, P.R. China

E-mail: zhiminsuo@163.com

*Contributed equally

Key words: gastric precancerous lesions, Helicobacter pylori, cyclooxygenase-2, inducible nitric oxide synthase precancerous lesion tissue, which may be associated with the occurrence and development of gastric precancerous lesions.

\section{Introduction}

Gastric cancer is one of the most common clinical malignancies. The condition has a high incidence in China and can have a serious impact on human health (1). The pathogenesis of gastric cancer is currently believed to include multiple steps: Chronic atrophic gastritis, intestinal metaplasia of the gastric mucosa, atypical hyperplasia of the gastric mucosa and the eventual development of gastric cancer (2-4). These steps (chronic atrophic gastritis, intestinal metaplasia of the gastric mucosa and atypical hyperplasia of the gastric mucosa) are known as gastric precancerous lesions. Gastric precancerous lesions play an important cohesive role in the development of tumors (5). Helicobacter pylori $(\mathrm{Hp})$ is one of the main causative agents of chronic active gastritis. Recent studies have shown that $\mathrm{Hp}$ infection is an independent risk factor for intestinal metaplasia, which suggests that it may play an important role in the occurrence and development of gastric precancerous lesions (6-8).

The mechanism underlying the Hp-induced occurrence and development of gastric precancerous lesions has yet to be fully elucidated. Cyclooxygenase-2 (COX-2) is the rate-limiting enzyme in the synthesis of prostaglandins, and its expression level is low or non-existent in normal tissues (9). Studies have shown that COX-2 exhibits high expression in numerous tumor tissues, which may be closely associated with tumorigenesis and metastasis $(10,11)$. Inducible nitric oxide synthase (iNOS) is the main observation index of the inflammatory reaction, and high expression levels of the enzyme are indicative that an inflammatory reaction is ongoing (12). The aim of the present study was to analyze the effect of $\mathrm{Hp}$ infection on the expression levels of COX-2 and iNOS in gastric precancerous lesion tissue and in a cell line in vitro, and to explore the clinical significance of COX-2 and iNOS in the development of $\mathrm{Hp}$-induced gastric precancerous lesions.

\section{Subjects and methods}

General data. A total of 114 patients, who were diagnosed with gastric precancerous lesions by gastroscopy and pathological examination in the Henan University Huaihe Hospital 
(Kaifeng, China) between June 2010 and 2013, were included in this study. Pathological diagnosis was classified according to the diagnostic criteria formulated by the National Chronic Gastritis Conference (13). Among these patients, 57 were confirmed to be Hp-positive by Warthin-Starry silver staining and the rapid urease test, and they were classified into the observation group; the remaining 57 patients were Hp-negative and were referred to as the control group. The observation group comprised 32 males and 25 females who were aged $38-75$ years (mean age, $51.4 \pm 10.2$ years); there were 25 cases of chronic atrophic gastritis, 20 cases of intestinal metaplasia of the gastric mucosa and 12 cases of atypical hyperplasia of the gastric mucosa. In the control group, there were 35 males and 22 females (age range, 39-73 years; mean age, $50.1 \pm 10.9$ years); among these patients, there were 22 cases of chronic atrophic gastritis, 21 cases of intestinal metaplasia of the gastric mucosa and 14 cases of gastric mucosal atypical hyperplasia. No significant differences were found in the age, gender, pathological type or other indicators between the two groups $(\mathrm{P}>0.05)$. This study was conducted in accordance with the Declaration of Helsinki and with approval from the Ethics Committee of Henan University Huaihe Hospital. Written informed consent was obtained from all participants.

Collection of clinical specimens. All patients were examined by gastroscopy. Part of the obtained tissue was used for the extraction of total RNA, and part of tissue was used for the detection of COX-2 and iNOS levels.

Cell infection experiment in vitro. Normal human gastric mucosal GES-1 cells (American Type Culture Collection, Manassas, VA, USA) were cultured to the exponential growth phase in Dulbecco's modified Eagle's medium with $10 \%$ fetal bovine serum (Gibco-BRL, Grand Island, NY, USA), and then digested with $0.25 \%$ pancreatin. The cell concentration was adjusted to $4 \times 10^{5} / \mathrm{ml}$, and the cells were inoculated in a 12-well plate with $1 \mathrm{ml}$ per hole. Following $24 \mathrm{~h}$ of culture, when the cells were completely attached to the wall, $1 \times 10^{5}$ CFU Hp (Qicheng Industrial Co., Ltd, Shanghai, China) was added to the 12-well plate. Equal quantities of PBS were added to the control group wells. The cells were placed in a $37^{\circ} \mathrm{C} \mathrm{CO}_{2}$ incubator for $6,12,18$ and $24 \mathrm{~h}$, and then dissolved in TRIzol ${ }^{\circledR}$ (Life Technologies, Carlsbad, CA, USA) for the extraction of total RNA. The expression of COX-2 and iNOS was analyzed.

Reverse transcription-quantitative polymerase chain reaction (RT-qPCR). Biopsy tissue or HP-treated cells were placed in $1 \mathrm{ml}$ TRIzol solution and $200 \mu \mathrm{l}$ chloroform was added to the homogenate. The homogenate was agitated, mixed and placed on ice for $15 \mathrm{~min}$ to create a layered solution, which was then centrifuged at $15,000 \mathrm{x}$ g for $15 \mathrm{~min}$. The supernatant was transferred to $500 \mu 1$ isopropanol, mixed, placed on ice for $15 \mathrm{~min}$ and further centrifuged at $15,000 \mathrm{x} g$ for $10 \mathrm{~min}$ for RNA precipitation. The precipitate was then washed twice with pre-cooled $75 \%$ ethanol, prior to being dissolved in double-distilled water treated with diethylpyrocarbonate. The concentration of the sample was determined, and RNA was transcribed into cDNA using an
RT kit (Takara, Dalian, China) to create a template for the PCR.

According to the COX-2 and iNOS mRNA sequences from GenBank (http://www.ncbi.nlm.nih.gov/genbank/), the primers were designed as follows: COX2 forward, 5'-GAA TCATTCACCAGGCAAATTG-3' and reverse, 5'-TCTGTA CTGCGGGTGGAACA-3'; iNOS forward, 5'-ACAACAAAT TCAGGTACGCTGTG-3' and reverse, 5'-TCTGATCAATGT CATGAGCAAAGG-3'; $\beta$-actin forward, 5'-GCGGGAAAT CGTGCGTGAC-3' and reverse, 5'-CGTCATACTCCTGCT TGCTG-3'. Following the dilution of primer, the conditions for specificity and the annealing temperature were optimized The reaction system was prepared as follows: $10 \mu 12 \mathrm{X} \mathrm{SYBR}$ Green qPCR Master Mix (Takara), $1 \mu 1$ upstream primer and $1 \mu \mathrm{l}$ downstream primer $(10 \mu \mathrm{mol} / 1)$, and $1 \mu \mathrm{l} \mathrm{cDNA}$. The double-distilled water was supplemented to a final volume of $20 \mu \mathrm{l}$. Following centrifugation at $1,800 \mathrm{x} \mathrm{g}$, the reaction mixture was gathered at the bottom of the tube. PCR was performed according to the following reaction conditions: Predegeneration at $95^{\circ} \mathrm{C}$ for $30 \mathrm{sec}$; degeneration at $95^{\circ} \mathrm{C}$ for $3 \mathrm{sec}$; and annealing and extension at $60^{\circ} \mathrm{C}$ for $30 \mathrm{sec}$ for 25 cycles. The standard curve was constructed. Data were then directly read from the $\mathrm{ABI}^{\circledR} 7500$ real-time PCR instrument (Applied Biosystems, Milan, Italy).

Western blot analysis. A total of $100 \mu 1$ cell lysate was obtained from the gastric mucosa tissue in two groups of patients, and $1 \mu 1$ protease inhibitor was added. The homogenate was then placed on ice and left to stand for $30 \mathrm{~min}$, prior to centrifugation at $15,000 \mathrm{x} \mathrm{g}$ for $15 \mathrm{~min}$. The supernatant was removed and the concentration of protein was measured. Sample buffer (4X) was added and boiled using boiling water for $30 \mathrm{~min}$. The sample was added and centrifugation was performed following the addition of sample buffer at $15,000 \times \mathrm{g}$ for $10 \mathrm{~min}$. Electrophoresis was performed using $12 \%$ SDS-PAGE at an initial voltage of $80 \mathrm{~V}$. The voltage was adjusted to $120 \mathrm{~V}$ once the bromophenol blue had entered the gel. When the electrophoresis was complete, the sample was transferred to a polyvinylidene difluoride membrane. Blocking was performed using 5\% skimmed milk powder, and then the primary monocolonal mouse antibody against COX-2 (1:100; \#sc-29411; Santa Cruz Biotechnology, Inc., Santa Cruz, CA, USA) was added for overnight incubation. The membrane was then washed with PBS-Tween 20 (PBST) three times. The horseradish-peroxidase conjugated goat anti-rat secondary antibody (ZSGB-BIO, Beijing, China) was then added for $1 \mathrm{~h}$ of incubation at room temperature, prior to three further washes with PBST. The ECL luminous liquid (ZSGB-BIO) was added, the signal was detected and images were captured. $\beta$-actin was considered as a reference protein. The band intensity of the target protein was analyzed using Tocan 430 gray calculation software (Tocan Biotechnology Co., Shanghai, China), and the relative expression level of the target protein was calculated.

Analysis of iNOS activity. The activity of iNOS was analyzed using the Griess reaction principle. In brief, a small quantity of gastric mucosa was homogenized and then centrifuged at $15,000 \mathrm{x} \mathrm{g}$ for $5 \mathrm{~min}$. The supernatant was removed and an equal volume of Griess reaction liquid (Wako Pure Chemical 


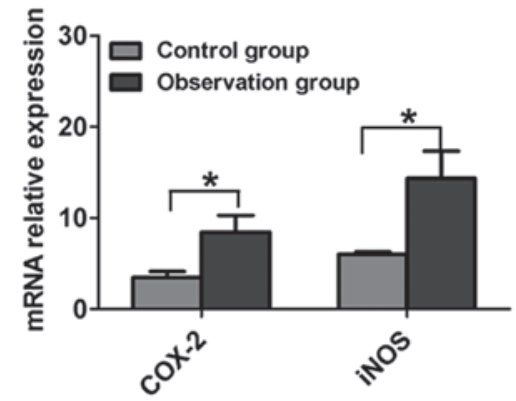

Figure 1. Comparison of COX-2 and iNOS mRNA expression in the gastric mucosa samples from the control and observation groups. Results are presented as the mean \pm standard deviation. ${ }^{*} \mathrm{P}<0.05$. COX-2, cyclooxygenase- 2 ; iNOS, inducible nitric oxide synthase.
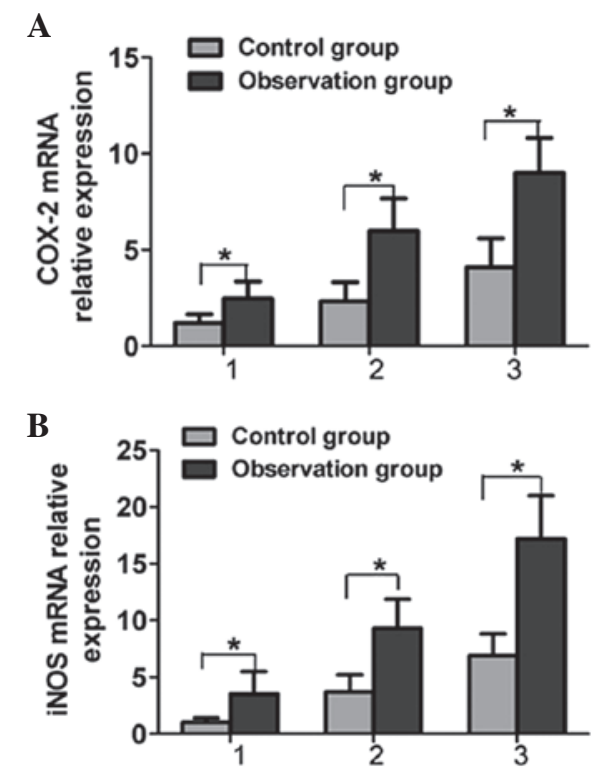

Figure 2. Comparison of (A) COX-2 and (B) iNOS mRNA expression in different pathological types of gastric mucosa: 1, chronic atrophic gastritis; 2 , gastric intestinal metaplasia; 3, atypical hyperplasia. Results are presented as the mean \pm standard deviation. ${ }^{*} \mathrm{P}<0.05$. COX-2, cyclooxygenase- 2 ; iNOS, inducible nitric oxide synthase.

Industries Ltd., Osaka, Japan) was added and mixed fully, and the mixture was then left to stand at room temperature for $15 \mathrm{~min}$. The optical density was analyzed at a wavelength of $540 \mathrm{~nm}$ by microplate reader. The NO level in the supernatant was calculated according to the results of standard curve to reflect the biological activity of iNOS.

Observation indexes. The mRNA and protein levels of COX-2 and iNOS in the gastric mucosa samples from the control and observation groups were analyzed. Furthermore, the changes in the mRNA and protein levels of COX-2 and iNOS in the GES-1 cells were examined at different time-points following treatment by $\mathrm{Hp}$.

Statistical analysis. All data were analyzed using SPSS 13.0 statistical software (SPSS Inc., Chicago, IL, USA). The measurement data are presented as the mean \pm standard deviation and were compared using the Student's t-test, $\mathrm{P}<0.05$ was considered to indicate a statistically significant difference.
A
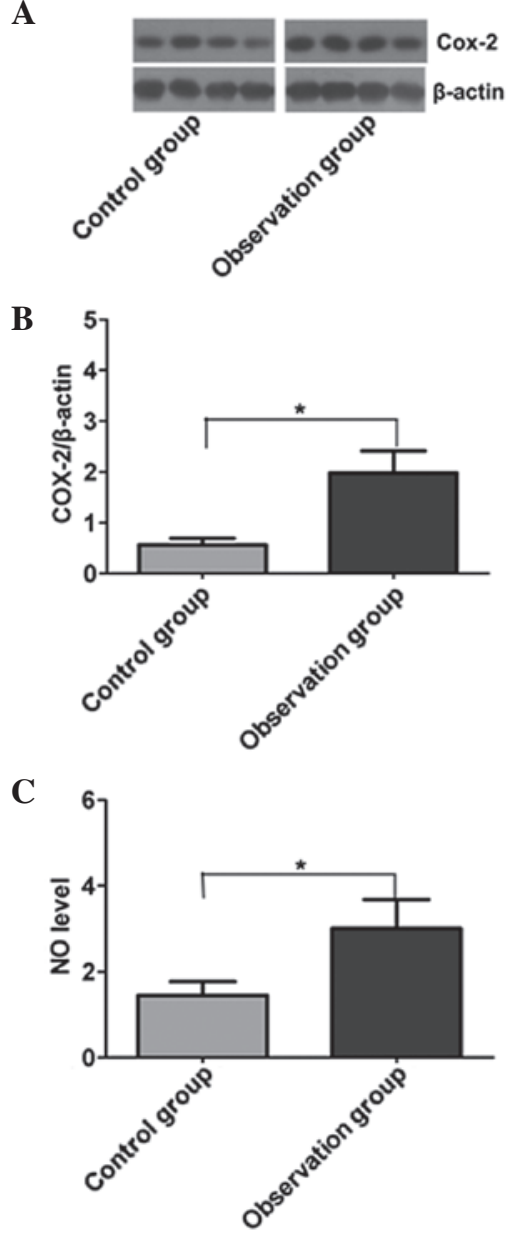

Figure 3. Comparison of the protein levels of COX-2 and iNOS in the two groups. (A) COX-2 expression, as detected by western blotting. (B) Quantitative analysis of COX-2 expression. (C) NO expression. Results are presented as the mean \pm standard deviation. "P $<0.05$. COX-2, cyclooxygenase-2; NO, nitric oxide.

\section{Results}

Comparison of COX-2 and iNOS mRNA levels in the gastric mucosa of the two groups. An RT-qPCR method was utilized to determine the COX-2 and iNOS levels in the gastric mucosa. The stability of the system was high, and the repeatability and melting curve were good. The results for the analysis of COX-2 and iNOS mRNA in the two gastric mucosa groups are shown in Fig. 1. Compared with the control group, the COX-2 and iNOS mRNA levels in the gastric mucosa of the patients in the observation group were significantly increased, and the difference was statistically significant $(\mathrm{P}<0.05)$ (Fig. 2). The COX-2 and iNOS mRNA levels in the gastric mucosa of the patients in the observation group with different pathological types were significantly higher than those in the control group $(\mathrm{P}<0.05)$.

Comparison of COX-2 and $\mathrm{CNOS}$ protein levels in the gastric mucosa of the two groups. Western blotting was used to analyze the changes in COX-2 protein expression in the gastric mucosa in the two groups, and quantitative correction was performed through the internal reference protein, $\beta$-actin (Fig. 3A). The expression level of COX-2 in the observation 
A

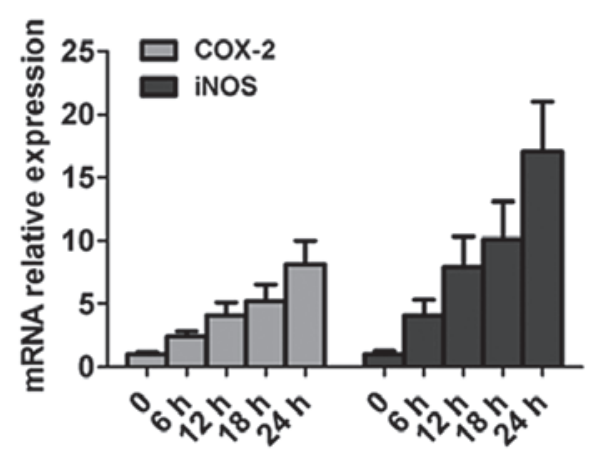

B

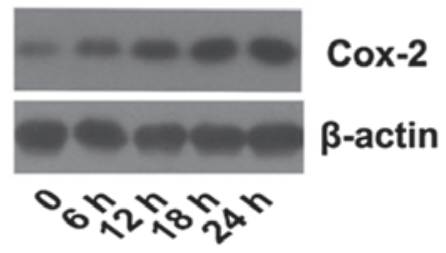

C

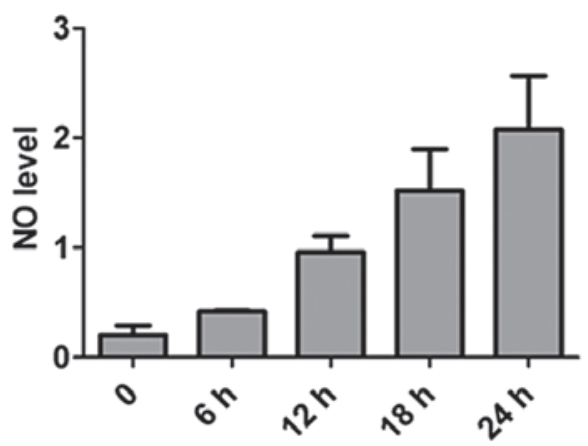

Figure 4. Effect of Helicobacter pylori on COX-2 and iNOS in normal gastric mucosa cells. (A) mRNA levels of COX-2 and iNOS. (B) Protein expression levels of COX-2, as detected by western blotting. (C) Expression levels of NO. Results are presented as the mean \pm standard deviation. COX-2, cyclooxygenase-2; NO, nitric oxide.

group was significantly higher than that in the control group $(\mathrm{P}<0.05)$. The NO (the reaction product of iNOS) level in the gastric mucosa was determined by the Griess reaction; the level of $\mathrm{NO}$ in the gastric mucosa of the observation group was significantly higher than that in the control group $(\mathrm{P}<0.05)$ (Fig. 3B).

Effect of Hp on COX-2 and iNOS mRNA expression in gastric mucosa cells. The infection process of $\mathrm{Hp}$ was simulated using normal gastric mucosa cells in vitro, and the changes in cellular COX-2 and iNOS mRNA were analyzed (Fig. 4). Compared with the negative control group, the expression levels of COX-2 and iNOS mRNA in the cells stimulated by Hp were significantly increased $(\mathrm{P}<0.05)$, and the changes showed a time-dependent manner. This suggested that Hp infection could stimulate cells to produce higher levels of COX-2 and iNOS.

\section{Discussion}

Gastric precancerous lesions are a type of histopathological change in the gastric mucosa and are closely associated with gastric cancer. The formation of precancerous lesions is an important stage in the transformation process from normal gastric mucosa to gastric carcinoma, including dysplasia and intestinal metaplasia. Studies have shown that the infection of the normal gastric mucosa with Hp can lead to chronic atrophic gastritis, intestinal metaplasia and dysplasia; however the molecular mechanism underlying these Hp-induced processes is still not completely understood at present $(14,15)$. COX-2 is an essential enzyme for the synthesis of prostaglandin, and is also the key rate-limiting enzyme in the initial step of prostaglandin synthesis. COX-2 is generally produced when the body suffers stimulation, and is involved in the inflammatory response (16). Studies have found an abnormal expression of COX-2 protein in several types of tumor tissues, which suggests that it may be involved in the tumorigenesis and the development and metastasis of cancer $(17,18)$. iNOS is generally produced by macrophages or monocytes and is the main sign of an inflammatory reaction. iNOS can aggravate the injury to the gastric mucosa. By promoting the imbalance between the proliferation and apoptosis of epithelial cells and promoting tumor angiogen- esis, iNOS is involved in the occurrence of gastric cancer and gastric lesions. iNOS production is the early main event in the development of gastric cancer $(19,20)$.

In the present study, RT-qPCR was initially utilized to analyze the mRNA levels of COX-2 and iNOS. The results showed that the gastric mucosal mRNA levels of COX-2 and iNOS were increased significantly in the patients with Hp-positive gastric precancerous lesions compared with those in the patients with $\mathrm{Hp}$-negative lesions. This indicated that $\mathrm{Hp}$ stimulation resulted in abnormal transcription of COX-2 and iNOS mRNA in the gastric mucosa of patients. The expression levels of COX-2 and iNOS protein were analyzed using western blotting. The analysis showed that the expression level of COX-2 protein in Hp-positive gastric cancer tissues was significantly higher than that in Hp-negative patients. Since NO is the main product of iNOS, measurement of NO was used to reflect the expression level of iNOS. The results showed that the NO level in Hp-positive gastric cancer tissues was significantly higher than that in the Hp-negative gastric cancer tissues. These results suggested that $\mathrm{Hp}$ infection may stimulate the gastric mucosa, resulting in an abnormal activation of COX-2 and iNOS transcription and translation. COX-2 and iNOS are the main inflammatory indexes, which indicated that Hp infection aggravated inflammation levels in the gastric mucosa. The present study showed that chronic inflammation was closely associated with tumorigenesis.

In order to explain the universality of this phenomenon, Hp was used to infect normal human gastric mucosal cells in vitro. The result showed that, with the extension of the infection time, the expression levels of COX-2 and iNOS increased significantly compared with the levels in unstimulated cells. This result was consistent with the detection results for the clinical sample. In addition, it was found that a correlation existed between the expression levels of COX2 and iNOS in gastric cancer and the pathological types of gastric precancerous lesions. The expression levels of COX-2 and iNOS increased significantly in the gastric mucosal tissues of severe pathological types, suggesting that serious chronic inflammation in the gastric mucosa was associated with a higher chance of gastric cancer.

In conclusion, the levels of COX-2 and iNOS were increased significantly in the mucosa of patients with gastric precancerous lesions infected by Hp. The expression of COX-2 
and iNOS was correlated with the pathological grading in patients; therefore, anti-Hp therapy should be performed to reduce the level of inflammation in the gastric mucosa and reverse the development of the disease.

\section{References}

1. Siegel R, Naishadham D and Jemal A: Cancer statistics, 2012. CA Cancer J Clin 62: 10-29, 2012.

2. Falt P, Hanousek M, Kundrátová E and Urban O: Precancerous conditions and lesions of the stomach. Klin Onkol 26 Suppl: S22-S28, 2013 (In Czech).

3. Rugge M, Capelle LG, Cappellesso R, Nitti D and Kuipers EJ: Precancerous lesions in the stomach: from biology to clinical patient management. Best Pract Res Clin Gastroenterol 27: 205-223, 2013.

4. Zorzetto V, Maddalo G, Basso D and Farinati F: Immunotherapy for gastric premalignant lesions and cancer. Immunotherapy 4: 587-599, 2012.

5. Watari J and Miwa H: Precancerous lesions of the stomach. Nihon Shokakibyo Gakkai Zasshi 107: 1759-1769, 2010 (In Japanese).

6. Rizzato C, Kato I, Plummer M, et al: Risk of advanced gastric precancerous lesions in Helicobacter pylori infected subjects is influenced by $\mathrm{ABO}$ blood group and cag A status. Int J Cancer 133: 315-322, 2013.

7. Kato I, Canzian F, Plummer M, et al: Polymorphisms in genes related to bacterial lipopolysaccharide/peptidoglycan signaling and gastric precancerous lesions in a population at high risk for gastric cancer. Dig Dis Sci 52: 254-261, 2007.

8. Deng X, Liu ZW, Wu FS, Li LH and Liang J: A clinical study of weining granules in the treatment of gastric precancerous lesions. J Tradit Chin Med 32: 164-172, 2012.

9. Greenhough A, Smartt HJ, Moore AE, Roberts HR, Williams AC, Paraskeva $\mathrm{C}$ and Kaidi A: The COX-2/PGE2 pathway: key roles in the hallmarks of cancer and adaptation to the tumour microenvironment. Carcinogenesis 30: 377-386, 2009.
10. Chell S, Kaidi A, Williams AC and Paraskeva C: Mediators of PGE2 synthesis and signalling downstream of COX-2 represent potential targets for the prevention/treatment of colorectal cancer. Biochim Biophys Acta 1766: 104-119, 2006.

11. Chu AJ, Chou TH and Chen BD: Prevention of colorectal cancer using COX-2 inhibitors: basic science and clinical applications. Front Biosci 9: 2697-2713, 2004.

12. Janakiram NB and Rao CV: iNOS-selective inhibitors for cancer prevention: promise and progress. Future Med Chem 4: 2193-2204, 2012.

13. Digestive Disease Branch of Chinese Medical Association: National seminar consensus on chronic gastritis. Zhong Hua Xiao Hua Za Zhi 20: 199-201, 2000 (In Chinese).

14. Correa P and Houghton J: Carcinogenesis of Helicobacter pylori. Gastroenterology 133: 659-672, 2007.

15. Kato I, van Doorn LJ, Canzian F, et al: Host-bacterial interaction in the development of gastric precancerous lesions in a high risk population for gastric cancer in Venezuela. Int J Cancer 119: 1666-1671, 2006.

16. Zhang J, Luo J, Ni J, et al: MMP-7 is upregulated by COX-2 and promotes proliferation and invasion of lung adenocarcinoma cells. Eur J Histochem 58: 2262, 2014.

17. Jana D, Sarkar DK, Ganguly S, et al: Role of cyclooxygenase 2 (COX-2) in prognosis of breast cancer. Indian J Surg Oncol 5: 59-65, 2014.

18. Kim JM, Kim JS, Jung HC, Song IS and Kim CY: Up-regulation of inducible nitric oxide synthase and nitric oxide in Helicobacter pylori-infected human gastric epithelial cells: possible role of interferon-gamma in polarized nitric oxide secretion. Helicobacter 7: 116-128, 2002.

19. Li JB, Li XM and Zhan W: Expression of VEGF and iNOS protein in gastric cancer and precancerous lesions. Yi Bao Yu Fen Zi Mian Yi Za Zhi 27: 561-562, 2011 (In Chinese).

20. Szlachcic A, Krzysiek-Maczka G, Pajdo R, et al: The impact of asymmetric dimethylarginine (ADAMA), the endogenous nitric oxide (NO) synthase inhibitor, to the pathogenesis of gastric mucosal damage. Curr Pharm Des 19: 90-97, 2013. 